\title{
Wide Bandwidth Integrated 1-4 GHz Feed Development for VLBI and SKA
}

\author{
Jan Geralt Bij de Vaate \\ ASTRON \\ P.O. Box 2, 7990 AA Dwingeloo, The Netherlands \\ E-mail: vaate@astron.nI
}

Miroslav Pantaleev, Marianna Ivashina, Jian Yang, Michael Lindqvist

Onsala Space Observatory

SE-439 92 Onsala, Sweden

E-mail: miroslav.pantaleev@chalmers.se

\section{Frank Schäfer, Reinhard Keller, Walter Alef}

Max-Planck-Institut für Radioastronomie

Auf dem Hügel 69, D-53121 Bonn, Germany

E-mail: fschaefer@mpifr-bonn.mpg.de

\begin{abstract}
Wide-band single pixel feeds such as the wide-band Eleven feed developed in Europe through Onsala Space Observatory/Chalmers are rapidly increasing in maturity. Compared to the traditional octave bandwidth feeds, wide bandwidth feeds, such as the Eleven feed, will create the possibility of significantly larger instantaneous frequency coverage observations with existing radio telescopes, such as those used in the VLBI networks, and for future radio telescopes, such as the Square Kilometre Array. This Project is supported by RadioNet3, Developments In VLBI Astronomy (DIVA).
\end{abstract}

11th European VLBI Network Symposium \& Users Meeting

October 9-12, 2012

Bordeaux, France 


\section{Introduction}

Wide-band single pixel feeds such as the wide-band Eleven feed developed in Europe through Onsala Space Observatory / Chalmers are rapidly increasing in maturity. Compared to the traditional octave bandwidth feeds, wide bandwidth feeds will create the possibility of significantly larger instantaneous bandwidth observations.

The bandwidth for the DIVA ${ }^{1}$ feed will be $1-4 \mathrm{GHz}$ as minimum requirement. Within the SKA [1] program this will be applicable for the Wide Band Signal Pixel Feed (WBSPF) element of the Advanced Instrumentation Program (AIP). Prototype Eleven feeds demonstrated bandwidths of 1-10 GHz [2]. A 1-4 GHz feed will replace 2 octave band feeds, a 1-10 GHz feed would replace 4 octave band feeds. Besides operational benefits, increased survey speed by using a wide bandwidth (BW) feed will also be a significant cost saving. DIVA will therefore investigate the possibility of realising a $1-10 \mathrm{GHz}$ feed. The impact of a potential modest increase in noise temperature of the wide band receiver system will be mitigated, for many science cases, since the figure of merit, $B W^{*} A_{\text {eff }} / T_{\text {sys }}$, will be significantly improved with the use of a wide band single pixel feed.

New technologies will be used for the Low Noise Amplifiers (LNA), which promise excellent results for cryo-cooled amplifiers. Processes like 70nm mHEMTs from OMMIC and 50nm mHEMTs from IAF (Fraunhofer) are especially considered for this purpose. Research, design and evaluation of these technologies will focus on low power, wide bandwidth and very low noise temperatures.

\section{Wide bandwidth}

\subsection{Requirements}

The SKA currently considers two dish configurations, a prima focus design and an off-set Gregorian design. The (most likely) added cost of an off-set Gregorian is justified with the potential of lower spill-over. In [3] and [5] the two concepts are evaluated.

\subsection{Antenna design: the Eleven antenna feed}

The Eleven feed has a nearly constant beam width, a fixed phase center location, high aperture efficiency over a decade bandwidth with a compact and low-profiled structure [5]. Figure 1 shows the new circular Eleven feed of $1.3-14 \mathrm{GHz}$ and the measured aperture efficiency with its sub-efficiencies [6]. The predicted and measured system noise temperatures are plotted in Figure 2. The system noise model adopted in this analysis accounts for the antenna impedance noise mismatch with the LNAs as well as the external noise sources (thermal noise of the ground and surrounding trees).

${ }^{1}$ DIVA, Developments In VLBI Astronomy, is a Joint Research Activity of RadioNet3 


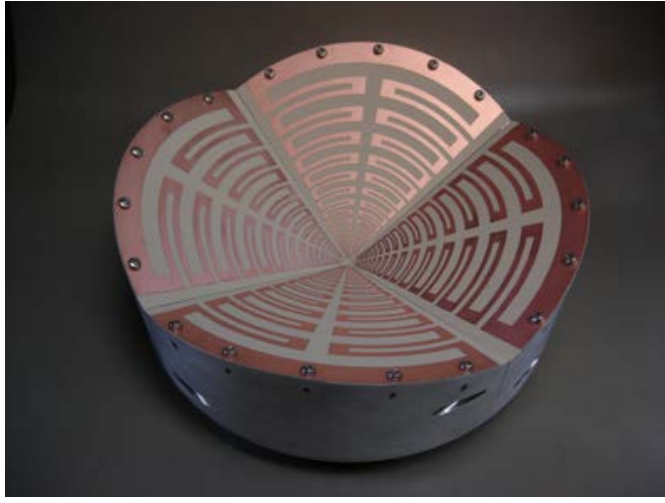

(a)

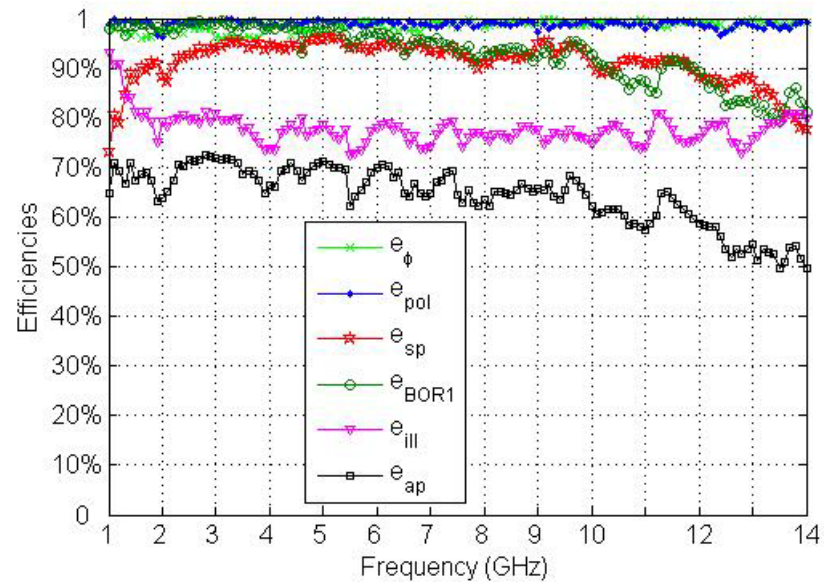

(b)

Figure 1. (a) The circular Eleven antenna feed and (b) the corresponding antenna efficiency contributions, as computed for the prime-focus reflector antenna with the subtended angle of $120^{\circ}$ based on the measured radiation function.

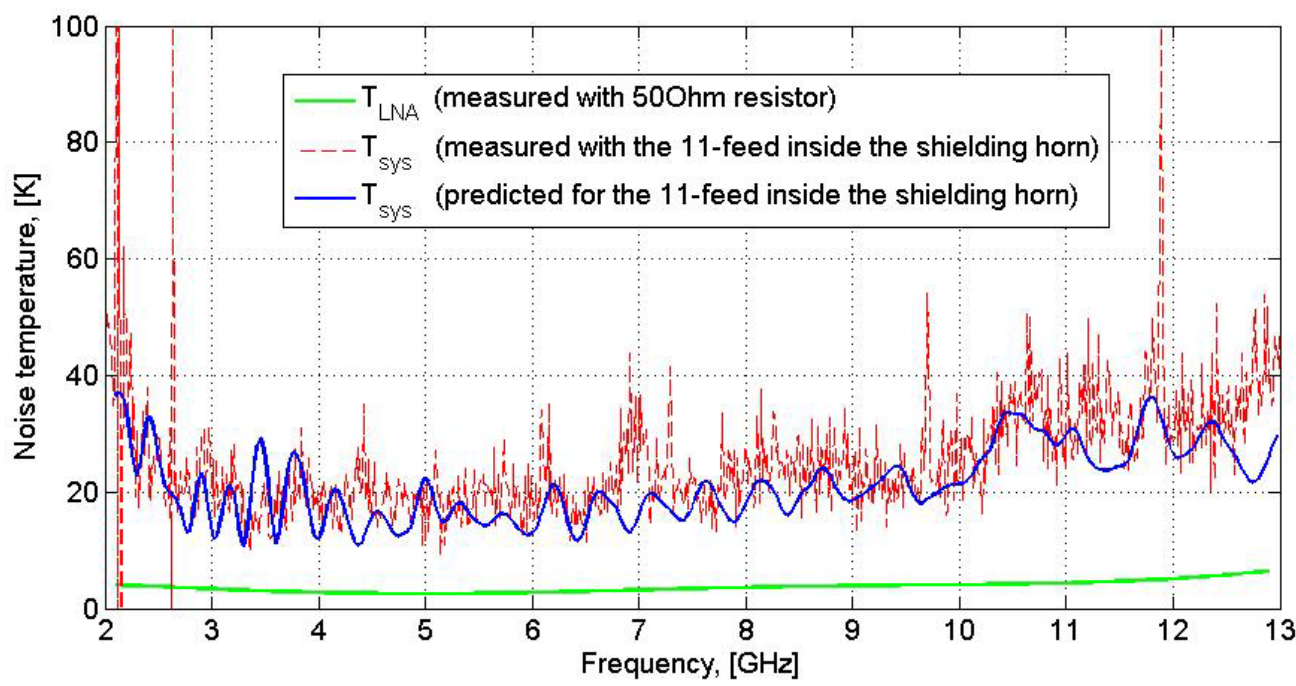

Figure 2. The modelled and measured system noise temperatures of the Eleven antenna feed receiver, when antenna is located on the ground and pointing towards the sky.

The results in Figure 2 are consistent with a receiver noise contribution for our measured hardware, which after excluding ground pick-up effects is $<10 \mathrm{~K}$. In conclusion, we can see that: (1) the developed system model works well for predicting the system noise temperature of the integrated antenna-receiver system; to the authors' best knowledge, it provides the best agreement with measured temperatures thus far presented for the Ultra Wide Bandwidth (UWB) feed system within the SKA community; (2) the data collected are consistent with very low receiver noise for an LNA coupled to an Eleven feed.

We show in Figure 3 our analysis of a scaled version of the circularized (1.3-14 GHz version) Eleven feed using the measured parameters of this feed combined with the CanadianUS SKA off-set Gregorian antenna design. The scaled feed was assumed to have a size of $30 \%$ larger to improve the low frequency performance. The results in Figure 3b show that our modeled feed/Gregorian reflector system has peak aperture efficiency over 83\% approaching 
that of the ideal horn feed optimized at each frequency. Over the range from 2 to $6 \mathrm{GHz}$ the aperture efficiency stays above $78 \%$ and from 1 to $2 \mathrm{GHz}$ it is above $68 \%$. Over the plotted bandwidth of factor 6, the antenna efficiency of the circular Eleven feed is only slightly lower than the aperture efficiency of the ideal feed (Fig. 3c) and on average higher than that of narrow angle octave horns. Table 1 shows the antenna temperature contribution from the Eleven feed at an elevation angle of $90^{\circ}$. The predicted performance of the ideal feed is based on the simulations of the feed patterns. In contrast to this ideal feed model, the antenna noise temperature of the Eleven feed was computed from the measured patterns of the feed. The antenna noise temperature $T \_s p$ of the Eleven feed is smaller than $10 \mathrm{~K}$ from 2 to $6 \mathrm{GHz}$ and has the minimum contribution of $6 \mathrm{~K}$ at around $5 \mathrm{GHz}$. The T_sp increases below $2 \mathrm{GHz}$ due to the pattern widening and above $7 \mathrm{GHz}$ due to the higher side-lobe levels of the feed pattern. When the feed is integrated inside the cryostat, the measured patterns become slightly narrower at low frequencies due to the effects of the cryostat window that starts acting as a part of the feed design at these frequencies. As the result, the spillover efficiency increases by about $0.2-0.5 \mathrm{~dB}$ (while the total antenna efficiency remains high due to the interplay between other efficiency components).

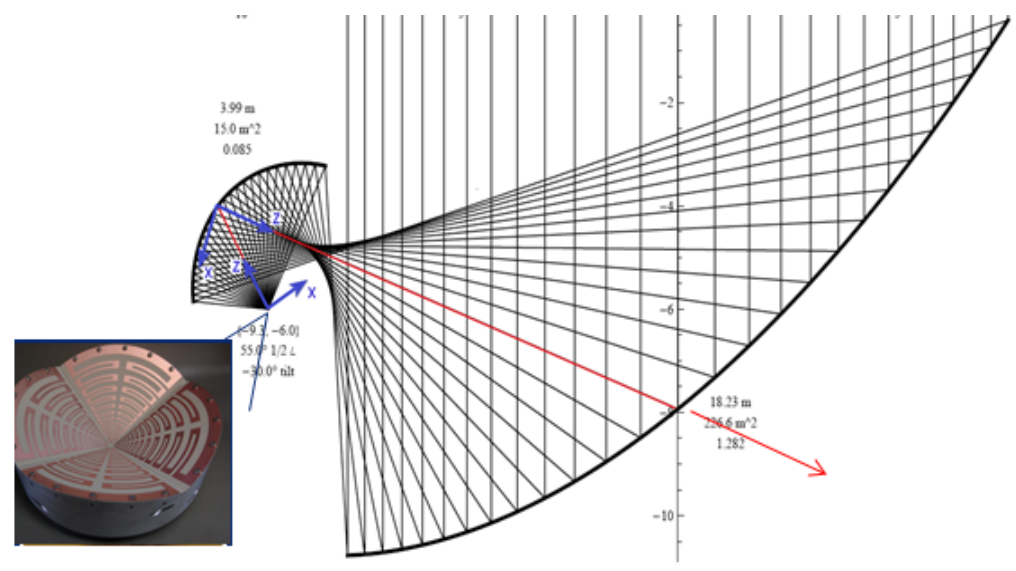

(a)

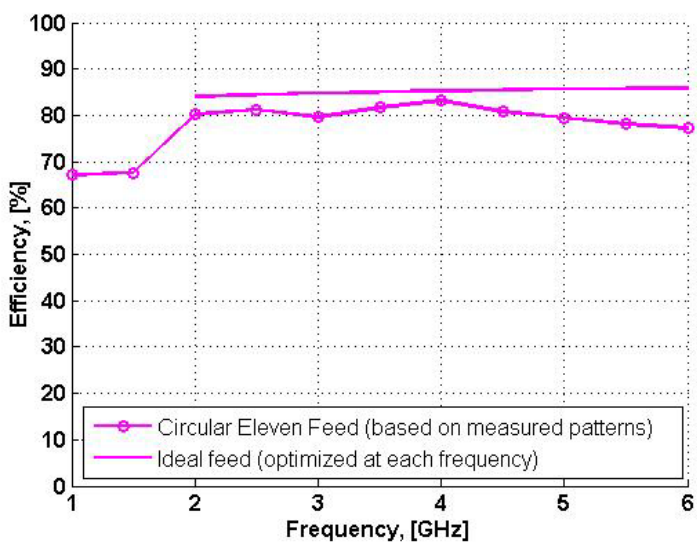

(b)

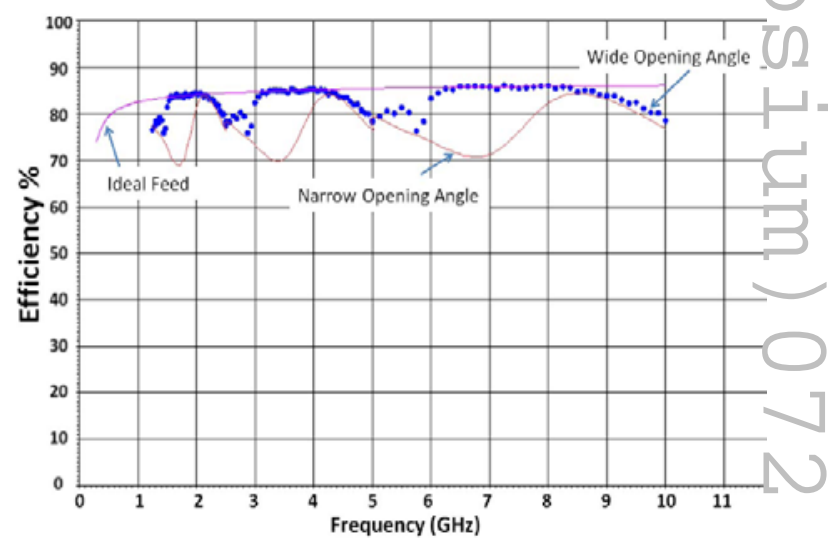

(c)

Figure 3. The predicted performance parameters of the 30\% scaled circularized Eleven feed combined with off-set Gregorian reflector optics (a) overall geometry, (b) antenna efficiency of Eleven feed compared with an ideal feed, (c) antenna aperture efficiency of octave band feeds and ideal feed optimized at each frequency. 


\begin{tabular}{|l|l|}
\hline \multicolumn{1}{|c|}{ At reference frequency $1.4 \mathrm{GHz}$} & \\
\hline Sky & 5 \\
\hline Spillover (elevation $=90^{\circ}$ ) & 10 \\
\hline Vacuum window & 1.5 \\
\hline Coupler & 1 \\
\hline LNA (connected to the Eleven antenna feed) & 7.5 \\
\hline Receiver & 0.5 \\
\hline Total Tsys & $\mathbf{2 5 ~ K}$ \\
\hline
\end{tabular}

Table 1. Realized noise temperature budgets for the Eleven feed in combination with the off-set Gregorian antenna system (subtended angle of the secondary reflector is $\left.110^{\circ}\right)$.

\subsection{Low Noise Amplifier design}

The large relative bandwidth requirements of $120 \%$ for $1-4 \mathrm{GHz}$ resp. $165 \%$ for $1-10 \mathrm{GHz}$ represent specifications for cryogenic LNA design that are not easy to fulfill. As a comparison, state of the art cryogenic receivers for radio astronomy nowadays cover the full bandwidth of standard rectangular waveguide bands which amounts to $40 \%$ or maybe even $67 \%$ for wideband receivers at lower frequencies $[7,8]$. A major decision for this project is whether to use singleended or differential LNAs. The antenna itself has differential ports natively, so different feeding topologies seem possible, employing various combinations of hybrids, baluns and single-ended / differential LNAs. This would call for minimum 2, maximum 8 cryogenic LNAs for 2 polarizations. Therefore, judging from complexity and serviceability of the instrument would also be in favor of differential LNAs at, or at least close to, the native feeding impedance of the antenna. This reasoning is naturally strongly influenced by comparison with the Allan Telescope Array [10] which is so far the only already existing instrument that pioneered the use of the wideband technologies discussed here. For this instrument wide-band single-ended and differential LNAs were developed and tested at CalTech [10], the latter being chosen for the receivers cooled to $\sim 60 \mathrm{~K}$ with Sterling type cryocoolers.

Although different LNA options will be evaluated within the project, we will focus on the conventional, single ended $50 \Omega$ design here; designs using SiGe HBT chip devices or a differential LNA prototype developed and fabricated at IAF will be discussed elsewhere.

\subsubsection{MMIC, $50 \Omega$ single-ended mHEMT design}

Choosing InP as a substrate material for HEMT devices is owing to the fact that the atomic layers needed to confine a 2-Dimensional Electron Gas (2DEG) and fabricated by MBE can be grown lattice-matched on InP. Another technological approach to achieve lattice-match for the device layers is to gradually adapt the substrates and device lattice constants by growing layers of subsequently varying stoichiometry, the resulting device is the so called metamorphic HEMT (mHEMT). From device physics the active part of a metamorphic HEMT is formed by an identical structure of atomic layers as compared to the InP based device which should result in similar noise performance. The advantage of the mHEMT is that it can be manufactured using GaAs as a substrate material which is less expensive and less brittle as compared to InP. This leads to both technological and economic advantages since it allows for processing on larger diameter wafers. The excellent noise performance of the Fraunhofer IAF mHEMT process at room temperature is well established [11]. Therefore, identifying this process as a candidate for 
fabrication of ultra low noise cryogenic devices for application in radio astronomy was a natural choice. Within several national and European cooperations, an evaluation of the cryogenic performance of IAF's mHEMT process with gate lengths of 50 and $100 \mathrm{~nm}$ was carried out. The first step consisted in establishing a cryogenic device model [12]. Subsequent testing of cryogenic LNAs designed using the model then indeed has showed that the IAF mHEMT process is competitive to the NGC InP process in terms of cryogenic noise performance [12].

The MMIC that we plan to test within the DIVA project is one of a series of cryogenic LNAs designed a few years ago as a first test to evaluate mHEMT LNA performance. Figure 4 shows the packaged MMIC alongside with a photo of the chip which is the first version fabricated using the $100 \mathrm{~nm}$ gate length process. Due to the low frequency of the lower bandpass edge and for improved noise performance, the first stage matching network is not realized on-chip but rather using an external substrate. Performance of the packaged LNA at room and cryogenic temperature is shown in Fig. 4. A subsequent redesign is planned to improve the bandwidth of the MMIC.
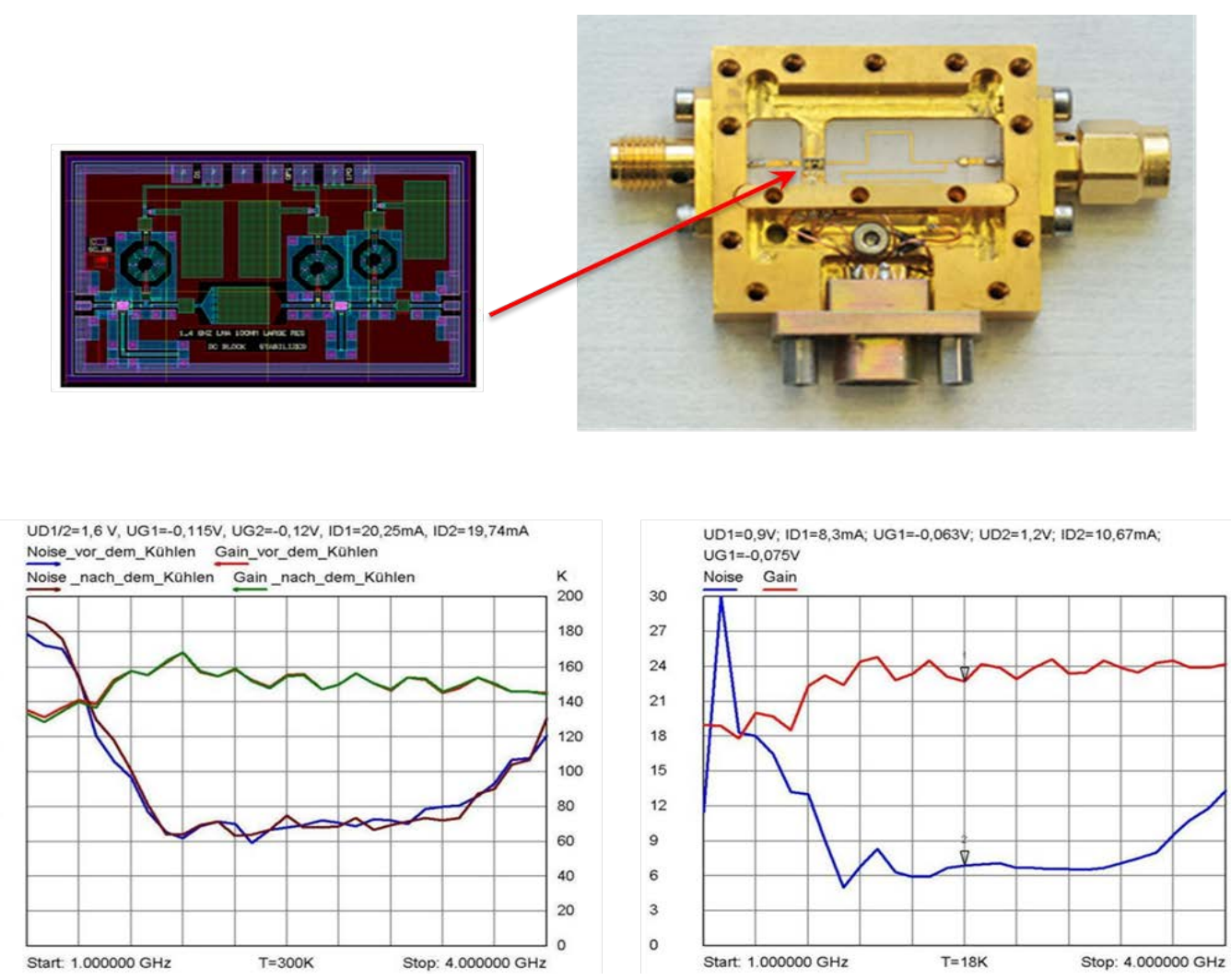

Figure 4. 1-4 GHz MMIC chip and packaged LNA (upper pictures), room temperature and cryogenic test results of the first version of the chip with 100nm gate length (lower plots).

\section{Conclusion}

Existing and new radio telescopes can be significantly improved when equipped with wide band feeds, not only in terms of bandwidth and operational performance but also in terms of the cost. The Eleven feed has a high potential of fulfilling these requirements. A close integration of antenna and LNA development is essential to achieve best performance. 


\section{Acknowledgement}

The research leading to these results has received funding from the European Commission Seventh Framework Programme (FP/2007-2013) under grant agreement No. 283393 (RadioNet3). Some of the authors (MPIfR) received funding from Fraunhofer/MPG cooperation funds within the "Kryo mHEMT" project. The authors thank the Fraunhofer IAF for providing information and data on their differential LNA MMIC.

\section{References}

[1] P. E. Dewdney, P. J. Hall, R. T. Schilizzi, and T. J. L. W. Lazio, The Square Kilometre Array, Proceedings of the IEEE, vol. 97, no. 8, pp. 1482-1496, 2009.

[2] J. Yang, X. Chen, N. Wadefalk, and P.-S. Kildal, Design and realization of a linearly polarized Eleven feed for 1-10 GHz, IEEE Antennas and Wireless Propagation Lett., vol. 8, pp. 64-68, 2009.

[3] M. V. Ivashina, R. Bakker, J. G. Bij de Vaate, O. A. Iupikov, M. Arts, J. Dekker, and A. van Ardenne, An Axi-Symmetric Segmented Composite SKA Dish Design: Performance and Production Analysis, Asian Pacific Microwave Conference, Melbourne, Dec. 2011.

[4] M. Pantaleev, J. Yin, M. Ivashina, and J. Conway, Final Report of Eleven Feed Project: Development of Broadband Cryogenic Frontend Prototype for the SKA, SKA memo 144, 2012.

[5] J. Yang, M. Pantaleev, P.-S. Kildal, B. Klein, Y. Karadikar, L. Helldner, N. Wadefalk, and C. Beaudoin, Cryogenic 2-13 GHz Eleven feed for reflector antennas in future wideband radio telescopes, IEEE Trans. on Antennas and Propagation, vol. 59, no. 6, pp. 1918-1934, 2011.

[6] J. Yin, J. Yang, M. Panteleev, and L. Helldner, A circular Eleven feed with significantly improved aperture efficiency over 1.3-14 GHz, 6th Eur. Conf. on Antennas Propagat. (EuCAP2012), Prague, 26-30 March 2012.

[7] R. Perley, et al., The Expanded Very Large Array, Proceedings of the IEEE, vol. 97, no. 8, pp. 1448-1462, 2009.

[8] R. Lehmensiek, and I. P. Theron, L-Band Feed Horn and Orthogonal Mode Transducer for the KAT-7 Radio Telescope, IEEE Trans. on Antennas and Propagation, vol. 59, no.6, pp 18941901, 2011.

[9] J. Welch, et al., The Allen Telescope Array: The First Widefield, Panchromatic, Snapshot Radio Camera for Radio Astronomy and SETI, Proceedings of the IEEE, vol. 97, no. 8, pp. 14381447, 2009.

[10] N. Wadefalk, and S. Weinreb, Noise testing for the active and passive baluns for the ATA, ATA memo \#67, 2004. Available online at: http://www.ral.berkeley.edu/ata/memos/memo67.pdf.

[11] A. Leuther, et al., Metamorphic HEMT technology for low-noise applications, IEEE International Conference on Indium Phosphide \& Related Materials, 10-14 May 2009, pp. 188-191, 2009.

[12] M. Seelmann-Eggebert, F. Schäfer, A. Leuther, and H. Massler, A versatile and cryogenic mHEMT-model including noise, IEEE International Microwave Symposium. Piscataway, NJ, pp. 501-504, 2010.

[13] B. Aja, et al., 4-12 GHz and 25-34 GHz Cryogenic MHEMT MMIC Low Noise Amplifiers for Radio Astronomy, IEEE International Microwave Symposium, 2012. 